\title{
Magnetic Curves Associated to Killing Vector Fields in a Galilean Space
}

\author{
Muhittin Evren Aydin*
}

(Communicated by Johann DAVIDOV)

Abstract
In this paper, we completely classify the magnetic curves (also $N$-magnetic curves
with constant curvature) in a Galilean 3-space associated to a Killing vector field
$V=v_{1} \partial_{x}+v_{2} \partial_{y}+v_{3} \partial_{z}$ with $v_{1}, v_{2}, v_{3} \in \mathbb{R}$.
Keywords: Magnetic curve; Lorentz equation; Killing vector field; cylindrical helix; Galilean space.
AMS Subject Classification (2010): Primary: $53 Z 05$; Secondary: 53A35; 53B25.
${ }^{*}$ Corresponding author

\section{Introduction}

A closed 2-form $F$ on a complete Riemannian manifold $(M, g)$ is called a magnetic field. The Lorentz force of a magnetic background $(M, g, F)$ is the skew symmetric $(1,1)$-type tensor field $\Phi$ on $M$ satisfying

$$
g(\Phi(X), Y)=F(X, Y),
$$

for any $X, Y \in \Gamma(T M)$. Thus a trajectory described by a charged particle moving in a magnetic field $F$ (or a magnetic curve associated to $F$ ) is a smooth curve $\gamma$ on $M$ satisfying the Lorentz equation (Newton equation or Landau-Hall equation):

$$
\nabla_{\dot{\gamma}} \dot{\gamma}=\Phi(\dot{\gamma})
$$

where $\nabla$ is the Levi-Civita connection of $g$. Note that the Lorentz force is divergence free, $\operatorname{div} \Phi=0$.

The fact that the Lorentz force is skew symmetric yields a basic property of magnetic curves, i.e. the following conservation law: particles evolve with constant speed (and so with constant energy) along the magnetic trajectories. If the magnetic curve $\gamma$ has unit speed, then it is called a normal magnetic curve. In the sequel, and all over this paper we study only the unit speed curves.

The study of magnetic fields and their trajectories on Riemannian (semi-Riemannian) manifolds, situated at the interplay between physics and differential geometry, has great interest. First problem regarding this phenomenal field was treated on Riemannian surfaces (see e.g. [8, 27]), then in 3-dimensional context, on $\mathbb{E}^{3}$ [16], $\mathbb{E}_{1}^{3}$ [17], $\mathbb{S}^{3}$ [9], $\mathbb{S}^{2} \times \mathbb{R}[23]$ etc. For the study of the magnetic curves associated to magnetic fields on arbitrary dimensional Kähler, contact manifolds and Walker 3-manifolds, we also refer the reader to [1, 2, 7, 10, 12, 13, 17, 18, 21, 22, 25].

Our aim is to obtain certain parametric equations of the magnetic curves in the Galilean 3-space $\mathbb{G}_{3}$ which is one model of the real Cayley-Klein geometries. For this, in the present paper, we consider only the magnetic curves associated to the Killing vector field of the form $V=v_{1} \partial_{x}+v_{2} \partial_{y}+v_{3} \partial_{z}$ with $v_{1}, v_{2}, v_{3} \in \mathbb{R}$. We classify such magnetic curves (also $N$-magnetic curves with constant curvature) in $\mathbb{G}_{3}$.

Received : 23-December-2015, Accepted : 09-March-2016 


\section{Preliminaries}

The Galilean geometry is one model of the real Cayley-Klein geometries (for details see [27]), which has projective signature $(0,0,+,+)$. The absolute figure of the Galilean geometry is an ordered triple $\{\omega, f, I\}$, where $\omega$ is the ideal (absolute) plane, $f$ a line in $\omega$ and $I$ is the fixed elliptic involution of the points of $f$. Detailed properties of Galilean space may be found in [3]-[6], [15, 19, 20, 24, 26].

A plane is called Euclidean if it contains $f$, otherwise it is called isotropic, i.e., planes $x=$ const. are Euclidean, in particular the plane $\omega$. Other planes are isotropic. A vector $X=\left(x_{1}, x_{2}, x_{3}\right)$ is said to be non-isotropic (resp. isotropic) if $x_{1} \neq 0$ (resp. $x_{1}=0$ ).

The Galilean scalar product between two vectors $X=\left(x_{1}, x_{2}, x_{3}\right)$ and $Y=\left(y_{1}, y_{2}, y_{3}\right)$ is given by

$$
\langle X, Y\rangle_{\mathbb{G}}=\left\{\begin{array}{l}
x_{1} y_{1}, \quad \text { when } x_{1} \neq 0 \text { or } y_{1} \neq 0 \\
x_{2} y_{2}+x_{3} y_{3}, \text { when } x_{1}=y_{1}=0
\end{array}\right.
$$

The norm of the non-isotropic vector $X$ is defined by $\|X\|_{\mathbb{G}}=\left|x_{1}\right|$; in case when $X$ is isotropic, $\|X\|_{\mathbb{G}}=\sqrt{x_{2}^{2}+x_{3}^{2}}$. $X$ is called a unit vector if $\|X\|_{\mathbb{G}}=1$.

The cross product in the sense of Galilean space is (see [3])

$$
X \times_{\mathbb{G}} Y=\left\{\begin{array}{c}
\left(0,-\left|\begin{array}{ll}
x_{1} & x_{3} \\
y_{1} & y_{3}
\end{array}\right|,\left|\begin{array}{ll}
x_{1} & x_{2} \\
y_{1} & y_{2}
\end{array}\right|\right), \text { when } x_{1} \neq 0 \text { or } y_{1} \neq 0 \\
\left(\begin{array}{cc}
x_{2} & x_{3} \\
y_{2} & y_{3}
\end{array} \mid, 0,0\right), \quad \text { when } x_{1}=y_{1}=0 .
\end{array}\right.
$$

A smooth admissible curve $\gamma$ (without isotropic tangent vectors) can be parameterized in $\mathbb{G}_{3}$ by

$$
\gamma: I \subset \mathbb{R} \longrightarrow \mathbb{G}_{3}, s \longmapsto(s, y(s), z(s)),
$$

where $s$ is the Galilean invariant of the arc length on $\gamma(I)$.

For an admissible curve, the curvature $\kappa(s)$ and torsion $\tau(s)$ are respectively given by

$$
\kappa(s)=\|\ddot{\gamma}\|_{\mathbb{G}}=\sqrt{\ddot{y}(s)^{2}+\ddot{z}(s)^{2}}
$$

and

$$
\tau(s)=\frac{\operatorname{det}(\dot{\gamma}, \ddot{\gamma}, \dddot{\gamma})}{\kappa(s)^{2}} .
$$

Obviously, $\kappa(s) \neq 0, \forall s \in I$. Thus, the orthonormal trihedron $\{T, N, B\}$ in the sense of Galilean geometry becomes

$$
\begin{aligned}
T(s) & =\dot{\gamma}(s)=(1, \dot{y}(s), \dot{z}(s)), \\
N(s) & =\frac{1}{\kappa(s)}(0, \ddot{y}(s), \ddot{z}(s)), \\
B(s) & =\frac{1}{\kappa(s)}(0,-\ddot{z}(s), \ddot{y}(s)) .
\end{aligned}
$$

In the sequel, the Frenet equations may be expressed by

$$
\frac{d}{d s}\left[\begin{array}{l}
T \\
N \\
B
\end{array}\right]=\left[\begin{array}{ccc}
0 & \kappa & 0 \\
0 & 0 & \tau \\
0 & -\tau & 0
\end{array}\right]\left[\begin{array}{l}
T \\
N \\
B
\end{array}\right] .
$$

\section{Killing Magnetic Trajectories in $\mathbb{G}_{3}$}

A smooth vector field $V$ on a Riemannian manifold $(M, g)$ is a Killing vector field if the Lie derivative with respect to $V$ of the metric $g$ vanishes, i.e

$$
\mathcal{L}_{V} g=0 .
$$

A smooth vector field $V$ on $M$ is Killing if and only if it fulfills the Killing equation

$$
g\left(\nabla_{X} V, Y\right)+g\left(\nabla_{Y} V, X\right)=0
$$


for all $X, Y \in \Gamma(T M)$, where $\nabla$ is the Levi-Civita connection on $M$.

The 2-forms on 3-dimensional manifolds may be identified with the corresponding vector fields via the Hodge $\star$ operator and the volume form $d v_{g}$ of the manifold. Hence, the magnetic fields correspond to divergence-free vector fields; in particular, the Killing vector fields yield an important class of the so called Killing magnetic fields.

Note that, the cross product of two vector fields $X, Y$ on $M$ can be defined as

$$
g(X \times Y, Z)=d v_{g}(X, Y, Z),
$$

where $X, Y, Z \in \Gamma(T M)$. Let $F_{V}=\iota_{V} d v_{g}$ be the Killing magnetic field corresponding to the Killing vector field $V$, where $\iota$ denotes the inner product. Equivalently, $F_{V} \wedge{ }^{b} V=d v_{g}$, where $b: T M \longrightarrow T M^{*}$ is the musical isomorphism. Then, the Lorentz force of $F_{V}$ is (see e.g. $[9,16]$ )

$$
\Phi(X)=V \times X .
$$

Consequently, the relations $(1.2)$ and (3.1) lead to the Lorentz force of the magnetic background $\left(\mathbb{G}_{3},\langle,\rangle_{\mathbb{G}}, F_{V}\right)$ :

$$
\ddot{\gamma}=V \times_{\mathbb{G}} \dot{\gamma},
$$

where $V$ is a Killing vector field on $\mathbb{G}_{3}$ and $F_{V}$ the corresponding Killing magnetic field.

Let $\gamma$ be a curve in $\mathbb{G}_{3}$, parameterized by the arc length given in the coordinate form

$$
\gamma(s)=(s, y(s), z(s)), s \in I \subset \mathbb{R},
$$

where $y$ and $z$ are smooth functions satisfying the initial conditions:

$$
y(0)=y_{0}, \dot{y}(0)=Y_{0} \text { and } z(0)=z_{0}, \dot{z}(0)=Z_{0} .
$$

We give a classification of the normal magnetic trajectories associated to the Killing vector $V=v_{1} \partial_{x}+v_{2} \partial_{y}+v_{3} \partial_{z}$ in $\mathbb{G}_{3}, v_{1}, v_{2}, v_{3} \in \mathbb{R}$.

Theorem 3.1. Let $\gamma$ be a normal magnetic trajectory associated to the Killing vector $V=v_{1} \partial_{x}+v_{2} \partial_{y}+v_{3} \partial_{z}$ in $\mathbb{G}_{3}$, satisfying the initial conditions (3.4). Then $\gamma$ has one of the following forms:

(i) if $V$ is isotropic,

$$
\gamma(s)=\left(s, \frac{v_{3}}{2} s^{2}+Y_{0} s+y_{0},-\frac{v_{2}}{2} s^{2}+Z_{0} s+z_{0}\right) ;
$$

(ii) otherwise, the cylindrical helix on $S_{\mathbb{G}}^{1}(r) \times l$, where $S_{\mathbb{G}}^{1}(r)$ is a Euclidean circle in $\mathbb{G}_{3}$ with radius $r=\left\{\left(\frac{Z_{0}}{v_{1}}-\frac{v_{3}}{v_{1}^{2}}\right)^{2}+\right.$ $\left.\left(\frac{Y_{0}}{v_{1}}-\frac{v_{2}}{v_{1}^{2}}\right)^{2}\right\}^{\frac{1}{2}}$ and $l$ is a straight line given by $\left(s, \frac{v_{2}}{v_{1}} s+\left(y_{0}-\frac{Z_{0}}{v_{1}}+\frac{v_{3}}{v_{1}^{2}}\right), \frac{v_{3}}{v_{1}} s+\left(z_{0}+\frac{Y_{0}}{v_{1}}-\frac{v_{2}}{v_{1}^{2}}\right)\right)$, parameterized by

$$
\begin{aligned}
\gamma(s)= & \left(s, \frac{\left(Z_{0}-\frac{v_{3}}{v_{1}}\right)}{v_{1}} \cos \left(v_{1} s\right)+\frac{\left(Y_{0}-\frac{v_{2}}{v_{1}}\right)}{v_{1}} \sin \left(v_{1} s\right)+\frac{v_{2}}{v_{1}} s+\left(y_{0}-\frac{\left(Z_{0}-\frac{v_{3}}{v_{1}}\right)}{v_{1}}\right),\right. \\
& \left.\frac{\left(Z_{0}-\frac{v_{3}}{v_{1}}\right)}{v_{1}} \sin \left(v_{1} s\right)-\frac{\left(Y_{0}-\frac{v_{2}}{v_{1}}\right)}{v_{1}} \cos \left(v_{1} s\right)+\frac{v_{3}}{v_{1}} s+\left(z_{0}+\frac{\left(Y_{0}-\frac{v_{2}}{v_{1}}\right)}{v_{1}}\right)\right) .
\end{aligned}
$$

Proof. The normal magnetic trajectories $\gamma$ are the solutions of the Lorentz equation (3.2). We divide the proof in two cases:

Case 1. $V$ is isotropic. Using the Galilean cross product (2.1), we get

$$
\left\{\begin{array}{c}
\ddot{y}=v_{3}, \\
\ddot{z}=-v_{2} .
\end{array}\right.
$$

After considering the initial conditions (3.4) into (3.6), the normal magnetic trajectory $\gamma$ takes the form (3.5), which gives the statement (i). 
Case 2. $V$ is non-isotropic. Then it follows from (2.1) and (3.2) that

$$
\left\{\begin{array}{l}
\ddot{y}=v_{3}-v_{1} \dot{z} \\
\ddot{z}=v_{1} \dot{y}-v_{2}
\end{array}\right.
$$

We may formulate the Cauchy problem associated to system (3.7) and the initial conditions (3.4) as follows:

$$
\left\{\begin{array}{l}
\dddot{y}=-v_{1}^{2} \dot{y}+v_{1} v_{2} \\
\dddot{z}=v_{1} v_{3}-v_{1}^{2} \dot{z}
\end{array}\right.
$$

After solving (3.8), we derive

$$
\begin{aligned}
& y(s)=\frac{v_{2}}{v_{1}} s+\frac{\left(Z_{0}-\frac{v_{3}}{v_{1}}\right)}{v_{1}} \cos \left(v_{1} s\right)+\frac{\left(Y_{0}-\frac{v_{2}}{v_{1}}\right)}{v_{1}} \sin \left(v_{1} s\right)+\left(y_{0}-\frac{\left(Z_{0}-\frac{v_{3}}{v_{1}}\right)}{v_{1}}\right), \\
& z(s)=\frac{v_{3}}{v_{1}} s+\frac{\left(Z_{0}-\frac{v_{3}}{v_{1}}\right)}{v_{1}} \sin \left(v_{1} s\right)-\frac{\left(Y_{0}-\frac{v_{2}}{v_{1}}\right)}{v_{1}} \cos \left(v_{1} s\right)+\left(z_{0}+\frac{\left(Y_{0}-\frac{v_{2}}{v_{1}}\right)}{v_{1}}\right),
\end{aligned}
$$

which constitutes the normal magnetic curves we are looking for. It is straightforward to prove that $\gamma$ is a cylindrical helix wrapped around $S_{\mathbb{G}}^{1}(r) \times l$.

Example 3.1. Let $\gamma$ be a normal magnetic trajectory associated to the Killing vector $V=v_{2} \partial_{y}+v_{3} \partial_{z}$ in $\mathbb{G}_{3}$. It is then expressed by (3.5). Choosing $Y_{0}=5, y_{0}=1, Z_{0}=3, z_{0}=4$ and $s \in I=[0, \pi]$, the normal magnetic trajectories $\gamma$ becomes the curves in blue color for $v_{2}=v_{3}=0$, in green color for $v_{2}=v_{3}=1$ and in red color for $v_{2}=v_{3}=2$ as in Figure 1:

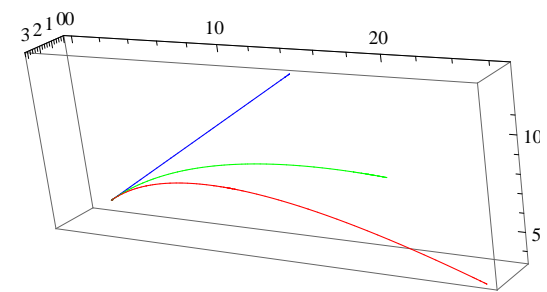

Figure 1. The magnetic trajectories associated to the Killing vectors $V \in\{(0,0,0),(0,1,1),(0,2,2)\}$.

\section{4. $N$-Magnetic ( $B$-Magnetic) Curves in $\mathbb{G}_{3}$}

In [11], Bozkurt et al. introduced a new kind of magnetic curves called $N$-magnetic curves ( $B$-magnetic curves) in oriented 3-dimensional Riemannian manifolds $(M, g)$ defined as follows:

Definition 4.1. Let $\gamma: I \subset \mathbb{R} \longrightarrow M$ be a curve in an oriented 3-dimensional Riemannian manifold $(M, g)$ and $F$ be a magnetic field on $M$. The curve $\alpha$ is an $N$-magnetic curve (respectively $B$-magnetic curve) if the normal vector field $N$ (respectively the binormal vector field $B$ ) of the curve satisfies the Lorentz force equation, i.e., $\nabla_{\dot{\gamma}} N=\Phi(N)=V \times N$ (respectively $\nabla_{\dot{\gamma}} B=\Phi(B)=V \times B$ ).

Several characterizations of $N$-magnetic curves (of $B$-magnetic curves as well) on $(M, g)$ were obtained in terms of the curvatures of the magnetic curve and the curvature of $(M, g)$ in [11].

In this section, we assume that the curve $\gamma$ has nonzero constant curvature $\kappa_{0}$ in order to obtain certain results.

Let consider the curve $\gamma$ in $\mathbb{G}_{3}$, parameterized by

$$
\gamma(s)=(s, y(s), z(s)),
$$

where $y$ and $z$ are smooth functions satisfying the initial conditions:

$$
y(0)=y_{0}, \dot{y}(0)=Y_{0}, \ddot{y}(0)=T_{0} \text { and } z(0)=z_{0}, \dot{z}(0)=Z_{0}, \ddot{z}(0)=U_{0} .
$$


We classify the $N$-magnetic curves with constant curvature $\kappa(s)=\kappa_{0} \neq 0$, corresponding to the Killing vector $V=v_{1} \partial_{x}+v_{2} \partial_{y}+v_{3} \partial_{z}$ in $\mathbb{G}_{3}, v_{1}, v_{2}, v_{3} \in \mathbb{R}$.

Theorem 4.1. Let $\gamma$ be a normal $N$-magnetic trajectory with constant curvature $\kappa_{0}$ associated to the Killing vector $V=v_{1} \partial_{x}+v_{2} \partial_{y}+v_{3} \partial_{z}$ in $\mathbb{G}_{3}$, satisfying the initial conditions (4.1). Then $\gamma$ is one of the following forms:

(i) If $v_{1}=v_{2}=v_{3}=0(V=0$, trivial Killing vector field $)$,

$$
\gamma(s)=\left(s, \frac{T_{0}}{2} s^{2}+Y_{0} s+y_{0}, \frac{U_{0}}{2} s^{2}+Z_{0} s+z_{0}\right) ;
$$

(ii) If $v_{1}=v_{2}=0$ and $v_{3} \neq 0$

$$
\gamma(s)=\left(s, Y_{0} s+y_{0}, \frac{U_{0}}{2} s^{2}+Z_{0} s+z_{0}\right)
$$

(iii) If $v_{1}=v_{3}=0$ and $v_{2} \neq 0$,

$$
\gamma(s)=\left(s, \frac{T_{0}}{2} s^{2}+Y_{0} s+y_{0}, Z_{0} s+z_{0}\right)
$$

(iv) If $v_{1}=0, v_{2} \neq 0, v_{3} \neq 0$,

$$
\gamma(s)=\left(s, \frac{U_{0}}{T_{0}} v_{2} s^{2}+Y_{0} s+y_{0}, \frac{U_{0}}{T_{0}} v_{3} s^{2}+Z_{0} s+z_{0}\right) ;
$$

(v) If $V$ is non-isotropic, $\gamma$ is the cylindrical helix on $S_{\mathbb{G}}^{1}(r) \times l$, where $S_{\mathbb{G}}^{1}(r)$ is a Euclidean circle in $\mathbb{G}_{3}$ with radius $r=\sqrt{\left(\frac{T_{0}}{v_{1}^{2}}\right)^{2}+\left(\frac{U_{0}}{v_{1}^{2}}\right)^{2}}$ and $l$ is a straight line given by $\left(s,\left(Y_{0}-\frac{U_{0}}{v_{1}}\right) s+\left(y_{0}+\frac{T_{0}}{v_{1}^{2}}\right),\left(Z_{0}+\frac{T_{0}}{v_{1}}\right) s+\left(z_{0}+\frac{U_{0}}{v_{1}^{2}}\right)\right)$ parameterized by

$$
\begin{aligned}
\gamma(s)= & \left(s, \frac{-T_{0}}{v_{1}^{2}} \cos \left(v_{1} s\right)+\frac{U_{0}}{v_{1}^{2}} \sin \left(v_{1} s\right)+\left(Y_{0}-\frac{U_{0}}{v_{1}}\right) s+\left(y_{0}+\frac{T_{0}}{v_{1}^{2}}\right),\right. \\
& \left.\frac{-U_{0}}{v_{1}^{2}} \cos \left(v_{1} s\right)-\frac{T_{0}}{v_{1}^{2}} \sin \left(v_{1} s\right)+\left(Z_{0}+\frac{T_{0}}{v_{1}}\right) s+\left(z_{0}+\frac{U_{0}}{v_{1}^{2}}\right)\right) .
\end{aligned}
$$

Proof. Assume that $\gamma$ is a normal $N$-magnetic curve corresponding to the Killing vector $V=v_{1} \partial_{x}+v_{2} \partial_{y}+v_{3} \partial_{z}$ in $\mathbb{G}_{3}$. By the Definition 4.1, we have

$$
\nabla_{\dot{\gamma}} N=\Phi(N)=V \times_{\mathbb{G}} N,
$$

where $N=\frac{1}{\kappa_{0}}(0, \ddot{y}(s), \ddot{z}(s))$. If $v_{1}=0$, it follows from (4.2) that

$$
\left\{\begin{array}{l}
\dddot{y}=0, \\
\dddot{z}=0, \\
v_{2} \ddot{z}-v_{3} \ddot{y}=0 .
\end{array}\right.
$$

By considering the initial conditions into (4.1), we immediately derive the statements (i)-(iv).

In the case $v_{1} \neq 0$, by using the cross product in $\mathbb{G}_{3}$ and $(4.2)$, we get that

$$
\left\{\begin{array}{l}
\dddot{y}=-v_{1} \ddot{z} \\
\dddot{z}=v_{1} \ddot{y} .
\end{array}\right.
$$

By solving the Cauchy problem associated to system (4.4) and the initial conditions (4.1), we obtain

$$
\begin{aligned}
& y(s)=\frac{-T_{0}}{v_{1}^{2}} \cos \left(v_{1} s\right)+\frac{U_{0}}{v_{1}^{2}} \sin \left(v_{1} s\right)+\left(Y_{0}-\frac{U_{0}}{v_{1}}\right) s+\left(y_{0}+\frac{T_{0}}{v_{1}^{2}}\right), \\
& z(s)=\frac{-U_{0}}{v_{1}^{2}} \cos \left(v_{1} s\right)-\frac{T_{0}}{v_{1}^{2}} \sin \left(v_{1} s\right)+\left(Z_{0}+\frac{T_{0}}{v_{1}}\right) s+\left(z_{0}+\frac{U_{0}}{v_{1}^{2}}\right),
\end{aligned}
$$

which completes the proof. 
Remark 4.1. For a trivial magnetic field, $V=0$, i.e. in the case of vanishing Lorentz force, the magnetic curves are given by the trajectories of the charged particles moving freely, only under the influence of gravity. So the solutions of the Lorentz equation become the geodesics, which satisfy $\nabla_{\dot{\gamma}} \dot{\gamma}=0$. This fact is not valid for $N$-magnetic curves in $\mathbb{G}_{3}$.

Example 4.1. Let $\gamma$ be a normal $N$-magnetic trajectory associated to the trivial Killing vector $V=v_{2} \partial_{y}+v_{3} \partial_{z}$ in $\mathbb{G}_{3}$. From the statements (i)-(iv) of Theorem 4.1, choose $T_{0}=1, Y_{0}=3, y_{0}=4$ and $U_{0}=1, Z_{0}=2, z_{0}=1, I=[0,5]$. Hence the pictures of the $N$-magnetic trajectories are the curves in blue color, in green color, in red color and in black color when the Killing vector $V$ is equal to zero, $\partial z, \partial y$ and $\partial y+2 \partial z$, respectively, as in Figure 2:

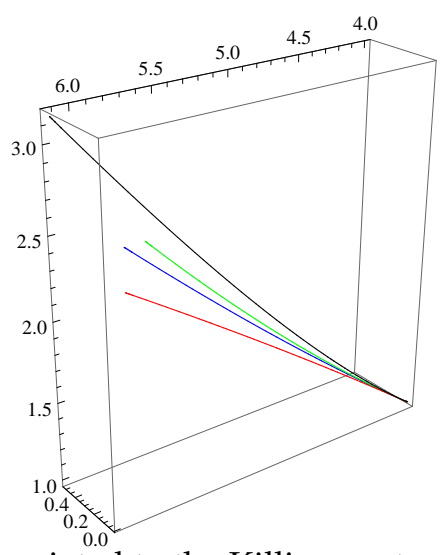

Figure 2. The $N$-magnetic trajectories associated to the Killing vectors $V \in\{(0,0,0),(0,1,0),(0,0,1),(0,1,2)\}$.

Remark 4.2. Similar results can be obtained by considering $B$-magnetic curves (instead of $N$-magnetic curves).

\section{References}

[1] Adachi, T., Kahler magnetic flow for a manifold of constant holomorphic sectional curvature. Tokyo J. Math. 18 (1995), no. 2, 473-483.

[2] Adachi, T., Kahler magnetic fields on a Kahler manifold of negative curvature. Diff. Geom. Appl. 29 (2011), 2-8.

[3] Ali, A.T., Position vectors of curves in the Galilean space $\mathbb{G}_{3}$. Matematički Vesnik. 64 (2012), no. 3, 200-210.

[4] Aydin, M.E. and Ergut, M., The equiform differential geometry of curves in 4-dimensional Galilean space $\mathbb{G}_{4}$. Stud. Univ. Babes-Bolyai Math. 58 (2013), no. 3, 393-400.

[5] Aydin, M.E., Ogrenmis, A.O. and Ergut, M., Classification of factorable surfaces in the pseudo-Galilean space. Glas. Mat. Ser. III. 50 (2015), no. 70, 441-451.

[6] Aydin, M.E., Mihai, A., Ogrenmis, A.O. and Ergut, M., Geometry of the solutions of localized induction equation in the pseudo-Galilean space. Adv. Math. Phys. vol. 2015, Article ID 905978, 7 pages, 2015. doi:10.1155/2015/905978.

[7] Bao, T. and Adachi, T., Circular trajectories on real hypersurfaces in a nonflat complex space form. J. Geom. 96 (2009), 41-55.

[8] Barros, M., Romero, A., Cabrerizo, J. L., and Fernandez, M., The Gauss-Landau-Hall problem on Riemannian surfaces. J. Math. Phys. 46 (2005), no. 11, 1-15.

[9] Barros, M., Cabrerizo, J. L., Fernandez, M., and Romero, A., Magnetic vortex filament flows. J. Math. Phys. 48 (2007), no. 8, 1-27.

[10] Bejan, C.-L. and Dructua-Romaniuc, S.L., Walker manifolds and Killing magnetic curves. Diff. Geom. Appl. 35 (2014), 106-16. 
[11] Bozkurt, Z., Gok, I., Yayli, Y. and Ekmekci, F.N., A new approach for magnetic curves in Riemannian manifolds. J. Math. Phys. 55 (2014), no. 5, 1-12.

[12] Cabrerizo, J. L., Fernandez, M., and Gomez, J.S., On the existence of almost contact structure and the contact magnetic field. Acta Math. Hungar. 125 (2009), no. 1-2, 191-199.

[13] Calvaruso, G., Munteanu, M.I. and Perrone, A., Killing magnetic curves in three-dimensional almost paracontact manifolds. J. Math. Anal. Appl. 426 (2015), no. 1, 423-439.

[14] Chen, B.-Y., Geometry of Submanifolds. M. Dekker. New York, 1973.

[15] Dede, M., Tubular surfaces in Galilean space. Math. Commun. 18 (2013), no. 1, 209-217.

[16] Dructua-Romaniuc, S.L. and Munteanu, M.I., Magnetic curves corresponding to Killing magnetic fields in $\mathbb{E}^{3}$. J. Math. Phys. 52 (2011), no. 11, 1-11.

[17] Dructua-Romaniuc, S.L. and Munteanu, M.I., Killing magnetic curves in a Minkowski 3-space. Nonlinear Anal., Real World Appl. 14 (2013), no. 1, 383-396.

[18] Dructua-Romaniuc, S.L., Inoguchi, J., Munteanu, M.I. and Nistor, A.I., Magnetic curves in Sasakian and cosymplectic manifolds. J. Nonlinear Math. Phys. 22 (2015), 428-447.

[19] Erjavec, Z., Divjak, B. and Horvat D., The general solutions of Frenet's system in the equiform geometry of the Galilean, pseudo-Galilean, simple isotropic and double isotropic space. Int. Math. Forum. 6 (2011), no. 17, 837-856.

[20] Kamenarovic, I., Existence theorems for ruled surfaces in the Galilean space. Rad Hazu Math. 456 (1991), no. 10, 183-196.

[21] Mohamed, J. and Munteanu, M.I., Magnetic curves on flat para-Kahler manifolds. Turkish J. Math. 39 (2015), no. 6, 963-969.

[22] Munteanu, M.I. and Nistor, A.I., Magnetic trajectories in a non-flat $\mathbb{R}^{5}$ have order 5. In: Van der Veken, J., Van de Woestyne, I., Verstraelen, L., Vrancken, L. (eds.) Proceedings of the Conference Pure and Applied Differential Geometry, PADGE 2012, pp. 224-231, Shaker Verlag Aachen (2013).

[23] Munteanu, M.I. and Nistor, A.I., The classification of Killing magnetic curves in $\mathbb{S}^{2} \times \mathbb{R}$. J. Geom. Phys. 62 (2012), 170-182.

[24] Ogrenmis, A.O., Ergut, M. and Bektas, M., On the helices in the Galilean Space $\mathbb{G}_{3}$. Iranian J. Sci. Tech. A. 31 (2007), no. A2, 177-181.

[25] Ozdemir, Z., Gok, I., Yayli, Y. and Ekmekci, F.N., Notes on magnetic curves in 3D semi-Riemannian manifolds. Turkish J. Math. 39 (2015), no. 3, 412-426.

[26] Oztekin, H., Special Bertrand curves in 4D Galilean space. Math. Probl. Eng. vol. 2014, Article ID 318458, 7 pages, 2014. doi:10.1155/2014/318458.

[27] Pavkovic, B.J. and Kamenarovic, I., The equiform differential geometry of curves in the Galilean space $\mathbb{G}_{3}$. Glasnik Mat. 22 (1987), no. 42, 449-457.

[28] Sunada, T., Magnetic flows on a Riemann surface. in: Proceedings of KAIST Mathematics Workshop, pp. 93-108 (1993).

\section{Affiliations}

MuHitTin EvREN AYDin

AdDress: Firat University, Dept. of Mathematics, 23119, Elazig-Turkey.

E-MAIL: meaydin@firat.edu.tr 